\title{
A healthy eating index to measure diet quality in pregnant women in Singapore: a cross-sectional study
}

Chad Yixian Han 1,2,3, Marjorelee Colega ${ }^{3}$, Elaine Phaik Ling Quah ${ }^{3}$, Yiong Huak Chan ${ }^{4}$, Keith M. Godfrey ${ }^{5}$, Kenneth Kwek ${ }^{6}$, Seang-Mei Saw ${ }^{7}$, Peter D. Gluckman ${ }^{3,8}$, Yap-Seng Chong ${ }^{3,9}$, and Mary Foong-Fong Chong ${ }^{3,10^{*}}$ on behalf of the GUSTO study group

\begin{abstract}
Background: There are limited tools to assess diet quality in pregnant women in an Asian population. A healthy eating index for pregnant women in Singapore (HEI-SGP) was developed and its association with maternal characteristics examined.

Methods: The HEI-SGP was adapted from the Healthy Eating Indices (HEI) and Alternate Healthy Eating Index for Pregnancy (AHEI-P) and modified accordingly to recommendations from the Singapore dietary guidelines for pregnant women. It included eight components to reflect the dietary adequacy and quality of food groups and two nutrient-based components to reflect nutrients to be taken in moderation. Total scores range from 0 to 100 . Study participants were from a mother-offspring cohort study - Growing Up in Singapore Towards healthy Outcomes (GUSTO), selected using criterion based sampling. Dietary intakes of these women were ascertained at 26-28 weeks of gestation using 24-hour recalls and 3-day food diaries.

Results: The HEI-SGP differentiated the diets qualitatively in the cohort of 955 women. The scores had a wide range of 12.6 - 94.3, with mean score of 52.4 (standard deviation 13.8) and were categorised by tertiles. Using one way ANOVA and chi-square tests, participants in the high tertile, compared to those in the middle and low tertiles, were more likely to meet recommendations for intakes of total fruits, whole fruits, total vegetables, dark green leafy and orange vegetables and dairy food groups $(p<0.001$ for all). Those in the low tertile had significantly higher percentage of energy from total fat $(p<0.001)$ and saturated fat $(p<0.001)$, and lower percentage of energy from protein $(p<0.001)$ compared to participants from the two higher tertiles. From adjusted multinomial logistic regression analyses, women with poorer diet quality tended to be younger (odds ratio $(\mathrm{OR})=0.94 ; 95 \%$ confidence interval (Cl): 0.90-0.97), belonged to the Malay ethnic group ( $\mathrm{OR}=2.54 ; 95 \% \mathrm{Cl}: 1.55-4.16)$, had lower household incomes $(\mathrm{OR}=2.00,95 \% \mathrm{Cl}: 1.03-3.87)$, were less educated $(\mathrm{OR}=1.96,95 \% \mathrm{Cl}: 1.19-3.25)$, single or had previous pregnancies (OR: 1.51; 95\%Cl 1.02, 2.24).
\end{abstract}

Conclusions: The HEI-SGP has shown to be useful for differentiating diet quality and may be used to identify women 'at risk' of poor diets during pregnancy and whom require early intervention.

Clinical Trial Registry: NCT01174875

Keywords: Healthy eating index, Diet quality, Diet quality indices, Pregnancy diet, Singapore pregnant women, Dietary guidelines

\footnotetext{
* Correspondence: mary_chong@sics.a-star.edu.sg

${ }^{3}$ Singapore Institute for Clinical Sciences, Brenner Centre for Molecular Medicine 30 Medical Drive, S117609 Singapore, Singapore

${ }^{10}$ Department of Paediatrics, Yong Loo Lin School of Medicine, National University of Singapore and National University Health System, 10 Medical Drive, S117597 Singapore, Singapore

Full list of author information is available at the end of the article
} 


\section{Background}

Diet quality indices were first developed to monitor a population's pattern of consumption and/or used to promote public health and education $[1,2]$. In recent years, these indices have been increasingly used to examine the relationships between the quality of diets and risk of various diseases $[3,4]$ and population groups, including pregnant women $[5,6]$.

Maternal nutrition is a key factor determining the healthy growth and development of the foetus and has implications on the cardiometabolic health of the offspring in later life [7]. To optimise the health of both mother and her offspring, many countries have dietary guidelines and recommendations developed specifically for pregnant women [8-11]. Adherence to these dietary guidelines has been used as a marker of diet quality. Several studies have adapted or developed diet quality indices to assess the diets of pregnant women. A study found that diet quality scores were negatively correlated to pre-conception and gestational BMI [6] while another found that diet quality declined significantly between study entry and 28 weeks gestation in overweight and obese pregnant women, and was maintained for 4 months post-partum [12].

Due to variations in dietary guidelines between different countries and cultural differences in diet, several diet quality indices for pregnant women have been developed. For example, an earlier study [13] validated the Healthy Eating Index (HEI), initially developed for healthy adults [14] by the U.S. Department of Agriculture (USDA) in 1995, for pregnant women. This HEI comprised ten components: fruit, vegetables, grains, milk, meat, total fat, saturated fat, cholesterol, sodium and variety of food choices. Another diet quality index for pregnant women is the Alternate Healthy Eating Index for Pregnancy (AHEI-P) [15], adapted from the Alternate Healthy Eating Index (AHEI) [16]. The latter had an additional component assessing use of multivitamin/mineral supplements containing iron, folate and calcium; micronutrients deemed important during pregnancy. Conceptually, the AHEI-P is similar to that of the HEI, except the former contains more nutrient-based components. The Dietary Quality Index for Pregnancy (DQI-P) [5], adapted from the diet quality index-revised (DQI-R) [17], is similar to the AHEI-P but contains fewer components. The Mediterranean diet scale for pregnant women (MDS-P) [18] was modified from the Mediterranean Diet adherence Scale (MDS) [19], which was originally used to measure diet adherence to dietary components that define a Mediterranean diet. The MDS-P is recommended for use in populations where the Mediterranean diet could be followed but may not be suitable for pregnant women living outside the Mediterranean region.
To our knowledge, this is the first study to date that investigated the use and relevance of available dietary quality indices for pregnant women in Singapore. The objective of this study was to develop a tool for measuring diet quality, which reflects the current local dietary guidelines and recommendations for pregnant women in Singapore. Additionally, we assessed associations of diet quality with maternal characteristics.

\section{Methods}

\section{Study population}

The present study was based on data from the Growing Up in Singapore Towards healthy Outcomes (GUSTO) mother-offspring cohort study [20]. Briefly, the GUSTO study is designed to investigate the effects of early life events on the risk of developing metabolic diseases later in life. From June 2009 to September 2010, pregnant women $(<14$ weeks' gestation) attending their antenatal care in Kandang Kerbau Women's and Children's Hospital $(\mathrm{KKH})$ and National University Hospital $(\mathrm{NUH})$ were recruited into the GUSTO study. Study participants recruited were Singapore citizens or permanent residents who were Chinese, Malay or Indian, had spouses of the same race, both whom had a homogenous ethnic parental background, aged between 18 and 50 years, with intention to deliver in $\mathrm{KKH}$ or $\mathrm{NUH}$, reside in Singapore for the next 5 years, and were willing to donate their cord, cord blood and placenta.

Women with significant health conditions such as type 1 diabetes mellitus or psychosis were excluded from the study. In addition, women with in vitro fertilization and multiple gestation pregnancies were also excluded. The Institutional Review Board of $\mathrm{KKH}$ and $\mathrm{NUH}$ approved the study. All participants gave written informed consent at time of recruitment.

\section{Data collection}

Trained personnel obtained information on demographics and socio-economic characteristics from all participants during recruitment. Participants were asked to attend clinic visits at 26-28 weeks of gestation after an overnight $(8 \mathrm{hr})$ fast, where fasting blood samples, anthropometric data and data relating to dietary and lifestyle characteristics (including cigarette smoking and alcohol consumption habits before and during pregnancy) were collected. Standing height and weight of participants were measured with a stadiometer (model 213; Seca, Hamburg, Germany) and digital scales (model 803; Seca) respectively and used for calculation of body mass indices. Dietary intake during pregnancy was assessed via an interview-administered 24-hour dietary recall (capturing intakes the previous day) and a 3-day food diary. The 24-hour dietary recall was collected by trained clinical staff using a 5-stage, multiple-pass interviewing technique 
[21]. Visual aids of food photographs and portion sizes were used during the interviews to facilitate accurate dietary data collection. The participants were also guided by these clinical staff in recording of the food diaries, which were done at home and returned at their next clinic visit. Nutrient analysis software (Dietplan, Forestfield software) and a local food composition database [22] were utilized for the nutrient analysis of these dietary records. Nutrient analyses of recipes were done with the nutrient analysis software for mixed dishes that were not available in the local database. Nutritional information of all other food items not available using the aforementioned methods, was obtained from the USDA national nutrient database or food labels. Fasting blood samples obtained from the participants were used to analyse plasma folate using the competitive electrochemiluminescence immunoassay on the ADVIA Centaur Immunoassay System. Between-run coefficients of variation (CV) for plasma measurements were $6.0 \%$ to $10.5 \%$ for folate samples between $1.8 \mathrm{ng} /$ $\mathrm{mL}$ to $9.8 \mathrm{ng} / \mathrm{mL}$.
Structure and development process of the Healthy Eating Index for Pregnant women in Singapore (HEI-SGP)

The Healthy Eating Index for Pregnant women in Singapore (HEI-SGP) was developed to examine diet quality in pregnant women in the present study. It is adapted from the Healthy Eating Indices (HEI) [14, 23, 24] and Alternate Healthy Eating Index for Pregnancy (AHEI-P) [15], modified accordingly to recommendations from the Singapore dietary guidelines for pregnant women [9]. For example, not all food-based and nutrient-based components from the HEI were included in the HEI-SGP as some of them were not part of the local dietary guidelines (e.g. seafood, seafood protein and fatty acids). A comparison of the food groups adopted by the aforementioned dietary indices can be found in the Additional file 1.

The HEI-SGP consists of a total of 11 components as shown in Table 1 . The first 8 components reflect the dietary adequacy and quality of food groups as recommended in the Singapore dietary guidelines for pregnant women [9]. The four adequacy components are total

Table 1 Healthy Eating Index for pregnant women in Singapore (HEI-SGP) components and standards for scoring

\begin{tabular}{|c|c|c|c|}
\hline Component & \multicolumn{3}{|c|}{ Score (points) } \\
\hline Total fruit & 0 & $\longleftrightarrow 5$ & $\geq 0.87$ serves $/ 1000 \mathrm{kcal}$ \\
\hline Whole fruit & 0 & $\longleftrightarrow 5$ & $\geq 0.43$ serves $/ 1000 \mathrm{kcal}$ \\
\hline Total vegetables & 0 & $\longleftrightarrow 5$ & $\geq 1.30$ serves/ $1000 \mathrm{kcal}$ \\
\hline $\begin{array}{l}\text { Dark green leafy and } \\
\text { orange vegetables }\end{array}$ & 0 & $\longleftrightarrow 5$ & $\geq 0.21$ serves/ $1000 \mathrm{kcal}$ \\
\hline $\begin{array}{l}\text { Total rice and } \\
\text { alternatives }\end{array}$ & 0 & $\Rightarrow 10$ & $\geq 2.60$ serves/ $1000 \mathrm{kcal}$ \\
\hline Whole grains & 0 & $\longrightarrow 10$ & $\geq 1.30$ serves/ $1000 \mathrm{kcal}$ \\
\hline Dairy & 0 & $\longrightarrow 10$ & $\geq 0.43$ serves/ $1000 \mathrm{kcal}$ \\
\hline Total protein foods & 0 & $\longrightarrow 10$ & $\geq 1.08$ serves/ $1000 \mathrm{kcal}$ \\
\hline $\begin{array}{l}\text { Use of antenatal } \\
\text { supplements }\end{array}$ & 0 & $\longleftrightarrow 5 \stackrel{\longrightarrow}{\longrightarrow} 10$ & $\begin{array}{l}\text { Consume supplements } \\
\text { containing iron, folate } \\
\text { and calcium }\end{array}$ \\
\hline Total fat* & 0 & $\longrightarrow 10$ & $\leq 30 \%$ of energy \\
\hline Saturated fat* & & 4 & $\leq 10 \%$ of energy \\
\hline
\end{tabular}

Total fruit includes all forms of fruits and $100 \%$ juice; Whole fruits including all forms except juice; Total vegetables include all forms of vegetables except starchy tuber vegetables like potatoes, yam except carrots. Dark green leafy and orange vegetables include vegetables such as mustard greens, bok choy and carrots. Total rice and alternatives includes grains, products made from flour, starchy tuber vegetables; Whole grains include wholemeal/wholegrain and products made from wholemeal flour; Dairy, includes all milk products, such as fluid milk, yoghurt, and cheese, and fortified soy beverages; Total protein foods includes meat, fish, beans and peas but excludes dairy; for antenatal supplements containing iron, folate and calcium: $0=$ supplements taken do not contain any of these 3 micronutrients; $5=$ supplements contained $1-2$ of these micronutrients; $10=$ supplements taken contained all 3 micronutrients.

*Zero score if total fat $>40 \%$ of energy and saturated fat $>20 \%$ of energy; $30 \%$ and $10 \%$ saturated fat is equivalent to $33.3 \mathrm{~g}$ and $11.1 \mathrm{~g}$ saturated fat per 1000 kcals respectively 
vegetables, total fruit, total rice and alternatives (known as brown rice and wholemeal bread in the guidelines), and total protein foods (known as meat and others in the guidelines), while the four components examining quality of the food groups are whole grains, dark green leafy and orange vegetables, whole fruit and dairy. Details of foods categorised under the specific components can be found in Additional file 1. Two more components are nutrient-based and reflect nutrients recommended to be taken in moderation. These are total fat and saturated fat, which have been recommended to be taken at $30 \%$ and $<10 \%$ of total energy intake respectively [9]. The final component, adapted from AHEI-P, reflects the degree of adherence to recommendations of antenatal supplements containing iron, folate and calcium as stipulated by the Singapore dietary guidelines for pregnant women [9]. The use of supplements containing these three micronutrients has been shown to be important in meeting the elevated requirement for these micronutrients during pregnancy [15]. The HEI-SGP assessed the reported use of antenatal supplements from 24-hour recalls as specific amounts of nutrients obtained from the supplements were not captured in the study.

\section{Weighting and scoring of HEI-SGP components}

Energy intakes and metabolic responses to pregnancy differ substantially between women [25-27]. We thus chose to represent intakes of foods and nutrients of participants using the energy density method. By using a density basis of recommended serve size per $1000 \mathrm{kcal}$ $[23,28]$, each individual's reported energy intake was adjusted for and the diet quality score was more comparable between individuals.

The HEI-SGP components were allocated sets of scores that measured compliance to the Singapore dietary guidelines for pregnant women. Following the approaches taken by Kennedy ET et al. for HEI [14], Rifas-Shiman SL et al. for AHEI-P [15] and McCullough ML et al. for AHEI [16], weighting of components was undertaken as below:

1. The food groups were first classed into three categories (as reflected in the Singapore Healthy Plate Model) [29] i.e. grains, meat \& others and fruit $\&$ vegetables, and each category allocated a total of 20 points. Under each category, the score was then divided to reflect adequacy and quality of the food groups met. For example, the total fruits and total vegetables components (reflecting total intake of fruits and vegetables) each contributed a maximum of 5 points to the total HEI-SGP score, while their quality components (reflecting quality of the fruit and vegetable consumed e.g. whole fruit vs fruit juices; dark green leafy and orange vegetables vs other types of vegetables) each contributed another maximum of 5 points, giving a total of 20 points.

2. Under the grains category, the total rice and alternatives component and whole grains component (reflecting quality of grains consumed) each contributed a maximum of 10 points to the total HEI-SGP score. Similarly, under the meat category, the total protein foods component and the dairy component (reflecting quality of protein) would each contribute a further 10 points each to the total HEI-SGP scores.

3. Participants were given the maximum score if they met the recommended intake levels and zero score if they did not; intermediate intakes were scored proportionately. Nutrient-based moderation components such as total and saturated fat were calculated from the 24-hour recalls and food diaries as $\%$ of total energy. Participants were given a maximum score of 10 if they adhered to the recommended intake levels (30\% and $10 \%$ of energy respectively), a zero score if they exceeded $40 \%$ of total energy intake for fat and $20 \%$ of total energy intake for saturated fat. Intermediate ranges (e.g. between $30 \%-40 \%$ ) were scored proportionately. A summary table of the scoring system is shown in Table 1.

4. The antenatal supplements component constituted another 10 possible points to reflect the importance of the use of antenatal supplements [30] in accordance with the Singapore dietary guidelines for pregnant women. Participants were given a score of 10 if the antenatal supplements they consumed contained all three micronutrients (iron, folate and calcium), a score of 5 if they contained one or two of these stated micronutrients, and a score of 0 if they contained none of these micronutrients [16].

5. The scores from the above components were summated to provide a raw HEI-SGP score, ranging from 0-90. The raw HEI-SGP scores were then converted to a scale of $0-100$, as per the original HEI. A detailed description of scoring a diet with the HEI-SGP can be found in Additional file 1.

\section{Statistical Analysis}

We used primarily the 24 hour recalls for dietary data as only a small subset of these participants $(n=260)$ provided the 3-day food diaries. As a sensitivity analysis, average HEI scores from 3-day food diaries from this subset of participants were used to validate the consistency of relationships between maternal characteristics and HEI-SGP (from 24-hour recalls) found in the main cohort. For the primary analyses, we excluded 17 women, whose 24-hour dietary recall gave an energy intake $<500$ or $>3500 \mathrm{kcal}$, leaving 955 women with informative data [31]. A 
flowchart of the subject included for this analysis can be found in Fig. 1. The mean and distribution of the scores of each HEI-SGP component within the cohort was examined and the scores were then categorised by tertiles. The relationship between nutrient intakes, \% recommended servings of food groups met, and scores by tertiles, were explored using one-way ANOVA. The relationship between maternal characteristics and HEI-SGP (tertiles) were first explored univariately, using one-way ANOVA for continuous variables and chi-square tests for categorical variables. Bonferroni post hoc tests on all possible pair wise comparisons were used if overall tests were significant. Next, the associations were examined by multinomial logistic regression and adjusted for total energy, age, BMI at 26 weeks of pregnancy, ethnicity, household income, education, marital status, cigarette smoking, and alcohol consumption. The analyses were made with reference to women with scores in the highest tertile. All analyses were conducted by using the SPSS statistical software version 16.0. A significance level of $\mathrm{p}<0.05$ was applied throughout.

\section{Results}

\section{Examining diet quality by HEI-SGP scores in tertiles}

In this cohort, the HEI-SGP scores covered a wide range from 12.6 to 94.3 , range of 86.1 with a mean (SD) of 52.4 (13.8), median of 52.6 and interquartile range 42.3-62.0. When the scores were categorised by tertiles, the score range for low, middle and high were 12.6-45.5, 45.6-58.6 and 58.7-94.3, respectively.

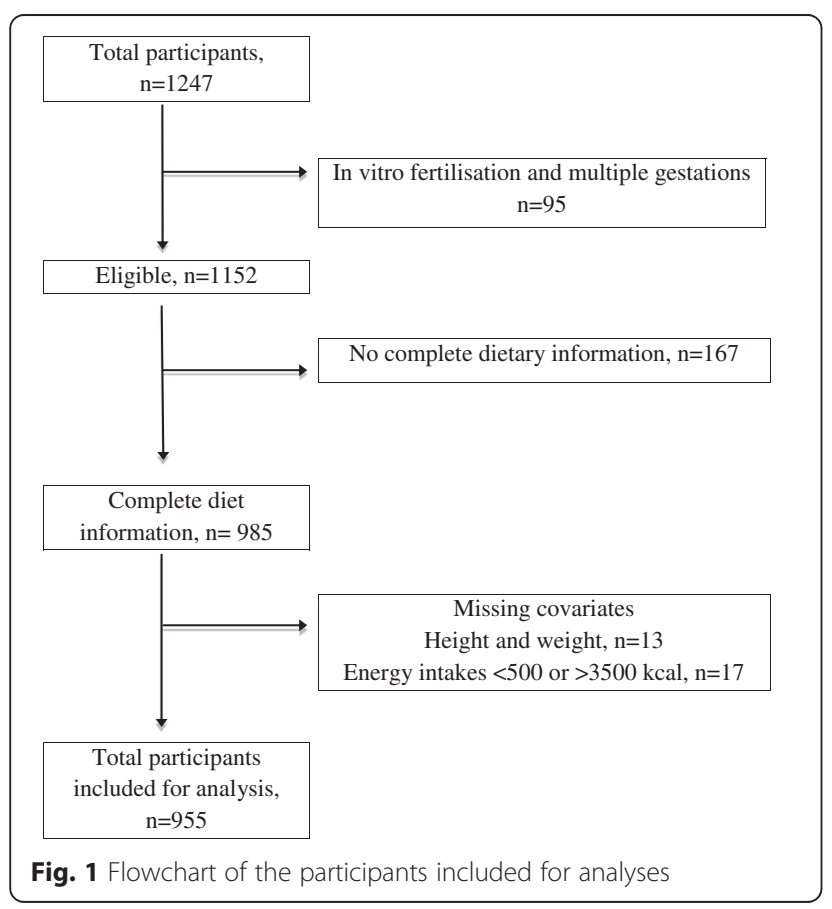

Table 2 illustrates that the HEI-SGP takes into account various components that captured variation in the quality of the diet. Examining the percentage of recommended servings of food groups (accordingly to Singapore dietary guidelines for pregnant women) met by participants, in general, the higher the score tertile, the higher percentages of recommended servings of each food group were met. Compared to those in the middle and low tertiles, participants in the high tertile were more likely to meet recommendations for intakes of total fruits, whole fruits, total vegetables, dark green leafy and orange vegetables and dairy food groups ( $\mathrm{p}<0.001$ for all). For total protein foods and total rice $\&$ alternatives, the percentage of participants eating recommended amounts was significantly higher in the high tertile when compared to the low tertile ( $\mathrm{p}=0.02$ and $\mathrm{p}<0.001$ respectively) but not when compared to the middle tertile. Significant differences in the percentages of participants achieving recommended servings were observed between the middle and low tertiles for most food groups ( $p<0.001$ for all). The percentage of recommended servings of whole grains and types of antenatal supplements met by participants in the high tertile was significantly higher compared to those in the middle or low tertile ( $\mathrm{p}<0.001$ for both), with no significant differences between the middle and low tertile.

To further test the internal consistency of this index, we compared nutrient intakes derived from the 24-hour recalls across the HEI-SGP tertiles. These included components in the HEI-SGP i.e. \% of energy from total fat and saturated fat as well as additional nutrients not in the HEI-SGP, including \% of energy from carbohydrate, protein, \% Recommended Dietary Intakes (RDI) for dietary calcium, dietary iron, and plasma folate concentrations. Participants in the high tertile had higher percentage of energy from carbohydrate compared to those in the low tertile $(\mathrm{p}<0.001)$. While there was no statistical significance between energy contributed from protein between the high and middle tertiles $(\mathrm{p}=0.316)$, participants in the low tertile had significantly lower percentage of energy from protein $(\mathrm{p}<0.001)$, compared to those participants from the other tertiles. Participants in the low tertile had the highest percentage of energy from total fat and saturated fat compared to women in the other tertiles $(\mathrm{p}<0.001$ for all). Participants in the high tertile had mean plasma folate concentrations that were significantly higher than participants in both the middle $(\mathrm{p}<0.001)$ and low tertile $(\mathrm{p}=0.002)$. For dietary calcium and iron, a statistically significant trend of increasing percentage of women meeting RDI is seen with higher tertiles $(\mathrm{p}<0.001$ for all).

Maternal characteristics and HEI-SGP of the GUSTO cohort The maternal characteristics of women $(n=955)$ from the GUSTO cohort study, by HEI-SGP tertiles are shown 
Table 2 Percentage recommended servings of food groups met and mean nutrient intakes in HEI-SGP score tertiles

\begin{tabular}{|c|c|c|c|c|}
\hline & \multicolumn{3}{|l|}{ HEI-SGP score ${ }^{a}$} & \multirow[b]{2}{*}{$P^{*}$} \\
\hline & $\begin{array}{l}\text { Low Tertile } \\
(n=319)\end{array}$ & $\begin{array}{l}\text { Middle Tertile } \\
(\mathrm{n}=316)\end{array}$ & $\begin{array}{l}\text { High Tertile } \\
(\mathrm{n}=320)\end{array}$ & \\
\hline \multicolumn{5}{|l|}{ Percentage recommended food group serves met per day } \\
\hline$\%$ Recommended total fruit serves per day ${ }^{a}$ & $23.1(36.5)^{* *}$ & $40.9(44.1)^{* *}$ & $72.0(38.7)^{* *}$ & $<0.001$ \\
\hline$\%$ Recommended whole fruit serves per day ${ }^{a}$ & $19.1(36.8)^{* *}$ & $38.8(47.0)^{* *}$ & $72.2(43.5)^{* *}$ & $<0.001$ \\
\hline$\%$ Recommended total vegetable serves per day ${ }^{a}$ & $35.3(29.5)^{* *}$ & $46.3(32.4)^{* *}$ & $62.7(32.3)^{* *}$ & $<0.001$ \\
\hline$\%$ Recommended dark green leafy and orange vegetable serves per day ${ }^{a}$ & $26.9(40.8) * *$ & $39.2(45.0)^{* *}$ & $60.0(45.1)^{* *}$ & $<0.001$ \\
\hline$\%$ Recommended total rice and alternatives serves per day ${ }^{a}$ & $82.5(20.5)^{* * * * * *}$ & $89.7(17.8)^{* *}$ & $91.7(14.6)^{* * *}$ & $<0.001$ \\
\hline \% Recommended whole grains per day & $3.4(14.5)^{* *}$ & $7.3(21.4)^{* * *}$ & $26.7(37.0)^{* * * * * *}$ & $<0.001$ \\
\hline$\%$ Recommended dairy serves per day ${ }^{a}$ & $36.2(39.1)^{* *}$ & $54.2(40.9)^{* *}$ & $69.6(36.0)^{* *}$ & $<0.001$ \\
\hline$\%$ Recommended total protein serve size per day ${ }^{a}$ & $77.7(29.3)^{* *}$ & $82.1(28.5)$ & $83.7(26.2)^{* *}$ & 0.02 \\
\hline$\%$ Recommended antenatal supplements containing all three micronutrients per day ${ }^{a}$ & $7.7(24.4)^{* *}$ & $13.1(31.5)^{* * *}$ & $33.4(44.3)^{* *, * * *}$ & $<0.001$ \\
\hline \multicolumn{5}{|l|}{ Nutrient intakes } \\
\hline Total energy $(\mathrm{kcal})^{\mathrm{a}}$ & $1924(594.9)^{* *}$ & $1795(566.2)^{* *}$ & $1852(507.9)$ & 0.014 \\
\hline Dietary carbohydrates (\% of total energy) ${ }^{a}$ & $47.5(8.7)^{* *}$ & $53.4(8.7)^{* *}$ & $55.1(7.1)^{* *}$ & $<0.001$ \\
\hline Dietary protein (\% of total energy) ${ }^{a}$ & $14.7(3.8)^{* * * * * *}$ & $15.8(4.0)^{* *}$ & $16.3(3.6)^{* * *}$ & $<0.001$ \\
\hline Dietary total fat (\% of total energy) ${ }^{a}$ & $37.8(7.0)^{* *}$ & $30.7(7.0)^{* *}$ & $28.6(5.7)^{* *}$ & $<0.001$ \\
\hline Dietary saturated fat (\% of total energy) ${ }^{a}$ & $16.1(4.6)^{* *}$ & $12.0(3.5)^{* *}$ & $10.8(2.9)^{* *}$ & $<0.001$ \\
\hline Dietary calcium as \% RDI (1000 mg) ${ }^{a}$ & $48.8(26.3)^{* *}$ & $56.8(29.8)^{* *}$ & $70.5(25.0)^{* *}$ & $<0.001$ \\
\hline Dietary iron as \% RDI (19 mg) ${ }^{a}$ & $59.9(23.7)^{* *}$ & $64.8(26.1)^{* *}$ & $80.7(22.3)^{* *}$ & $<0.001$ \\
\hline Plasma folate $(\mathrm{ng} / \mathrm{ml})^{\mathrm{a}}$ & $16.1(19.7)^{* *}$ & $15.3(9.7)^{* * *}$ & $20.9(21.5)^{* * * * * *}$ & $<0.001$ \\
\hline
\end{tabular}

*P-values obtained by one-way ANOVA tests for continuous variables

*****Numbers with similar superscript alphabets are statistically different, $p<0.05$

${ }^{a}$ Values reflect the mean (standard deviation) for continuous variables

${ }^{\mathrm{b}} \mathrm{HEI}-\mathrm{SGP}$ score range: low, 12.6-45.5; middle, 45.6-58.6; high 58.7-94.3

in Table 3. Pregnant women in the high HEI-SGP tertile tended to be older and had lower body mass index (BMI) than those in the middle or low tertile $(\mathrm{p}<0.001$ for all). Compared to women in the low tertile, those in the high HEI-SGP tertile also tended to be more highly educated $(p<0.001)$, had higher household incomes $(\mathrm{p}<0.001)$, were more likely to be married and living with their husbands $(\mathrm{p}=0.004)$, on their first pregnancy $(\mathrm{p}=0.061)$ and not smoked regularly before $(\mathrm{p}<0.001)$ and during pregnancy $(\mathrm{p}=0.005)$. Interestingly, a larger percentage of women who drank alcohol before pregnancy belonged to the high tertile, which could be a reflection of better economic status. Across different ethnicities, a higher percentage of Chinese women belonged to the high tertile, while a higher percentage of Malay women belonged to the low tertile. There was no significant difference in scores for working status, alcohol usage during pregnancy and moderate/strenuous exercise during pregnancy across tertiles. The relationships between the various maternal characteristics and HEI-SGP tertiles as aforementioned remained largely similar even when HEI scores were derived from 3-day food diaries from a subgroup of women (Additional file 1).
Adjusting for all covariates in a multivariate model (as shown in Table 4), the factors which predicted women belonging to the low HEI-SGP tertile were being younger $(\mathrm{OR}=0.94,95 \% \mathrm{CI}: 0.90-0.97)$, being of Malay ethnicity $(\mathrm{OR}=2.54,95 \% \mathrm{CI}$ : 1.55-4.16), having low household incomes of $<\mathrm{S} \$ 2000(\mathrm{OR}=2.00$, 95\%CI: 1.03 $3.87)$ and having an education level of secondary or less $(\mathrm{OR}=1.96,95 \% \mathrm{CI}: 1.19-3.25)$. In addition, women who had previous pregnancies had an increased likelihood (OR: 1.51 ; 95\%CI 1.02, 2.24) of belonging to the low tertile than women with first-time pregnancies.

\section{Discussion}

Our study developed a tool (HEI-SGP) for measuring diet quality in pregnant women in Singapore based on data from a 24-hour dietary recall categorised according to adherence to current local dietary guidelines and recommendations. The HEI-SGP was successful in quantitatively differentiating diet quality using one 24-hour dietary recall. Furthermore, we found differences in diet quality by maternal and socio demographic factors using the HEI-SGP. 
Table 3 Maternal characteristics by HEI-SGP in the GUSTO cohort study

\begin{tabular}{|c|c|c|c|c|}
\hline & \multicolumn{3}{|c|}{ HEI-SGP score ${ }^{a}$ GUSTO cohort } & \multirow[b]{2}{*}{$P^{*}$} \\
\hline & Low Tertile $(n=319)$ & Middle Tertile $(n=316)$ & High Tertile $(n=320)$ & \\
\hline Total energy $(\mathrm{kcal})^{\mathrm{b}}$ & $1924(594.9)$ & $1795(566.2)$ & $1852(507.9)$ & 0.014 \\
\hline Age $(\text { years })^{b}$ & $26.7(5.4)$ & $30.5(5.1)$ & $31.7(4.6)$ & $<0.001$ \\
\hline BMI 26 weeks $\left(\mathrm{kg} / \mathrm{m}^{2}\right)^{\mathrm{b}}$ & $26.3(4.6)$ & $26.8(4.7)$ & $25.4(3.6)$ & $<0.001$ \\
\hline \multicolumn{5}{|l|}{ Ethnicity $^{c}$} \\
\hline Chinese & $150(28.7)$ & $160(30.6)$ & $213(40.7)$ & \multirow[t]{3}{*}{$<0.00$} \\
\hline Malay & $121(47.8)$ & $92(36.4)$ & $40(15.8)$ & \\
\hline Indian & $48(26.8)$ & $64(35.8)$ & $67(37.4)$ & \\
\hline \multicolumn{5}{|l|}{ Gravidity $^{c}$} \\
\hline First pregnancy & $76(27.7)$ & $97(35.4)$ & $101(36.9)$ & \multirow[t]{2}{*}{0.061} \\
\hline Not first pregnancy & $243(35.7)$ & $219(32.2)$ & $219(32.2)$ & \\
\hline \multicolumn{5}{|l|}{ Marital status ${ }^{c}$} \\
\hline Single, not living with husband & $18(58.1)$ & $10(32.3)$ & $3(9.7)$ & \multirow[t]{2}{*}{0.004} \\
\hline Married, living with husband & $301(32.6)$ & $306(33.1)$ & $317(34.3)$ & \\
\hline \multicolumn{5}{|l|}{ Educational status ${ }^{c}$} \\
\hline Primary/Secondary & $116(40.7)$ & $103(36.1)$ & $66(23.2)$ & \multirow[t]{3}{*}{$<0.00$} \\
\hline Post-secondary & $133(38.2)$ & $114(32.8)$ & $101(29.0)$ & \\
\hline University and above & $70(21.7)$ & $99(30.7)$ & $153(47.5)$ & \\
\hline \multicolumn{5}{|l|}{ Work } \\
\hline No & $103(34.2)$ & $110(36.5)$ & $88(29.2)$ & \multirow[t]{2}{*}{0.131} \\
\hline Yes & $216(33.0)$ & $206(31.5)$ & $232(35.5)$ & \\
\hline \multicolumn{5}{|l|}{ Household income category $^{c}$} \\
\hline$<S \$ 2000$ & $62(46.6)$ & $45(33.8)$ & $26(19.5)$ & \multirow[t]{3}{*}{$<0.00$} \\
\hline S\$2000-5999 & $203(35.7)$ & $190(33.5)$ & $175(30.8)$ & \\
\hline$>S \$ 6000$ & $54(21.3)$ & $81(31.9)$ & $119(46.9)$ & \\
\hline \multicolumn{5}{|l|}{ Smoking during pregnancy ${ }^{c}$} \\
\hline No & $304(32.7)$ & $308(33.1)$ & $318(34.2)$ & \multirow[t]{2}{*}{0.005} \\
\hline Yes & $15(60.0)$ & $8(32.0)$ & $2(8.0)$ & \\
\hline \multicolumn{5}{|l|}{ Smoking regular before pregnancy ${ }^{c}$} \\
\hline No & $260(31.2)$ & $277(33.3)$ & $296(35.5)$ & \multirow[t]{2}{*}{$<0.00$} \\
\hline Yes & $59(48.4)$ & $39(32.0)$ & $24(19.7)$ & \\
\hline \multicolumn{5}{|l|}{ Alcohol use during pregnancy ${ }^{c}$} \\
\hline No & $311(33.3)$ & $310(33.2)$ & $314(33.6)$ & \multirow[t]{2}{*}{0.819} \\
\hline Yes & $8(40.0)$ & $6(30.0)$ & $6(30.0)$ & \\
\hline \multicolumn{5}{|l|}{ Alcohol usage before pregnancy ${ }^{c}$} \\
\hline No & $213(33.7)$ & $223(35.3)$ & $196(31.0)$ & \multirow[t]{2}{*}{0.044} \\
\hline Yes & $106(32.8)$ & $93(28.8)$ & $124(38.4)$ & \\
\hline \multicolumn{5}{|c|}{ Combined moderate and Strenuous Exercise ${ }^{c}$} \\
\hline No & $310(33.5)$ & $306(33.0)$ & $310(33.5)$ & \multirow[t]{2}{*}{0.963} \\
\hline Yes & $9(31.0)$ & $10(34.5)$ & $10(34.5)$ & \\
\hline
\end{tabular}

*P-values obtained by one-way ANOVA tests for continuous variables and chi-square tests for categorical variables.

${ }^{a} \mathrm{HEI}$-SGP score range: lowest, 12.6-45.5; middle, 45.6-58.6; highest 58.7-94.3

${ }^{b}$ Values reflect the mean (standard deviation) for continuous variables

'Values expressed as absolute numbers (percentage) for categorical variables; percentage may not add up due to rounding 
Table 4 Multivariate adjusted associations between maternal characteristics associated and HEI-SGP score

\begin{tabular}{|c|c|c|}
\hline & \multicolumn{2}{|c|}{ HEI-SGP score ${ }^{\mathrm{b}}$ GUSTO cohort } \\
\hline & \multicolumn{2}{|l|}{${ }^{\mathrm{a}}$ Adjusted model } \\
\hline & $\begin{array}{l}\text { Low Tertile } \\
(\mathrm{n}=319) \text { OR }(95 \% \mathrm{Cl})\end{array}$ & $\begin{array}{l}\text { Middle Tertile } \\
(\mathrm{n}=316) \text { OR }(95 \% \mathrm{Cl})\end{array}$ \\
\hline Total energy (log) & $5.64(1.58-20.05)^{* *}$ & $0.86(0.26-2.90)$ \\
\hline Maternal Age & $0.94(0.90-0.97)^{* *}$ & $0.96(0.92-0.99)^{*}$ \\
\hline $\mathrm{BMI}$ at 26 weeks & $1.03(0.99-1.08)$ & $1.07(1.03-1.12)^{* *}$ \\
\hline \multicolumn{3}{|l|}{ Ethnicity } \\
\hline Indian & $0.88(0.55-1.42)$ & $0.94(0.60-1.48)$ \\
\hline Malay & $2.54(1.55-4.16)^{* * *}$ & $1.75(1.06-2.89)^{*}$ \\
\hline Chinese & Ref & Ref \\
\hline \multicolumn{3}{|l|}{ Household Income } \\
\hline$<S \$ 2000$ & $2.00(1.03-3.87)^{*}$ & $1.17(0.61-2.25)$ \\
\hline$S \$ 2000-5999$ & $1.37(0.87-2.16)$ & $0.95(0.63-1.44)$ \\
\hline$>S \$ 6000$ & Ref & Ref \\
\hline \multicolumn{3}{|l|}{ Education Level } \\
\hline$\leq$ secondary & $1.96(1.19-3.25)^{* *}$ & $1.71(1.06-2.78)^{*}$ \\
\hline Post Secondary & $1.62(1.04-2.53)^{*}$ & $1.27(0.83-1.94)$ \\
\hline$\geq$ University & Ref & Ref \\
\hline \multicolumn{3}{|l|}{ Marital Status } \\
\hline Single & $3.31(0.91-12.1)$ & $2.31(0.60-8.90)$ \\
\hline Married & Ref & Ref \\
\hline \multicolumn{3}{|l|}{$\begin{array}{l}\text { Smoking before } \\
\text { pregnancy }\end{array}$} \\
\hline Yes & $0.91(0.49-1.69)$ & $0.89(0.47-1.68)$ \\
\hline No & Ref & Ref \\
\hline \multicolumn{3}{|l|}{$\begin{array}{l}\text { Smoking during } \\
\text { pregnancy }\end{array}$} \\
\hline Yes & $3.33(0.68-16.4)$ & $2.80(0.53-14.7)$ \\
\hline No & Ref & Ref \\
\hline \multicolumn{3}{|l|}{$\begin{array}{l}\text { Alcohol consumption } \\
\text { before pregnancy }\end{array}$} \\
\hline Yes & $0.94(0.63-1.39)$ & $0.75(0.51-1.09)$ \\
\hline No & Ref & Ref \\
\hline \multicolumn{3}{|l|}{$\begin{array}{l}\text { Combined moderate, } \\
\text { strenuous exercise }\end{array}$} \\
\hline Yes & $1.18(0.44-3.15)$ & $1.22(0.49-3.07)$ \\
\hline No & Ref & Ref \\
\hline \multicolumn{3}{|l|}{ Gravidity } \\
\hline First pregnancy & $1.51(1.02-2.24)^{*}$ & $0.97(0.67-1.41)$ \\
\hline Not first pregnancy & Ref & Ref \\
\hline
\end{tabular}

${ }^{*} \mathrm{P}<0.05 ; *$ * $\mathrm{P}<0.01 ;{ }^{* * * \mathrm{P}}<0.001$

${ }^{a}$ Adjusted for all characteristics simultaneously

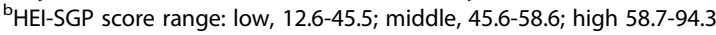

\section{Examining diet quality using HEI-SGP scores by tertiles}

We found that the mean percentages of recommended servings of each food group met by participants were significantly different across tertiles, demonstrating that the HEI-SGP was not dependent on a single or a few components of the diet and that the weightings used in deriving the score gave a broad representation of adherence to recommendations.

The consistent increase in percentage recommended servings met by the various food groups/component with increasing tertiles paralleled the increase in \% RDI met by the key vitamins and minerals, which suggests that using the food-based components was adequate to capture these important dietary constituents, without them being included in the HEI-SGP directly. The considerable lower proportion of energy intake from fats compared to carbohydrate and protein in participants of the high tertile reflected their close adherence to the dietary guidelines of the healthy plate, where carbohydraterich sources are recommended to form a larger proportion of the diet and fat consumption a smaller proportion.

\section{Maternal characteristics and HEI-SGP}

In this study, women in the low tertile had higher total energy intake than women in the other tertiles. This somewhat corresponds to the BMI at $26^{\text {th }}$ week gestation of the women, where BMI of the women in the lowest and middle tertile were significantly higher than women in the highest tertile. In contrast, previously reported data had shown that diet quality and total energy intake were positively correlated. Two separate studies found that higher energy intake in pregnant women with better quality Mediterranean diet (MD) [32, 33]. Dietary data from both cohorts used were collected using food frequency questionnaire (FFQ) to estimate variety and frequency of intake. Participants with greater variety and higher frequency of certain food would be deemed to have higher diet quality. However, this may also lead to higher energy intakes being calculated from the FFQ. For the present study, the aforementioned biasness was moderated by use of energy-adjusted intakes, a diet scoring system based on foods consumed per $1000 \mathrm{kcal}$. Consistent with three other studies [32-34], we found that older women were more likely to have a better diet quality compared to younger women. This may be because older women are likely to have planned pregnancies, and consequently more likely to eat more healthily to prepare for the pregnancy. Older women have been found to have better nutritional knowledge and better adherence to national dietary guidelines, and this may explain the better quality diets they have [35].

Socioeconomic status has also been observed to have a strong association with diet quality. Pregnant women who belonged to the category of " $>350 \%$ above poverty index" had significantly higher diet quality than those who belonged to the "185 to $<350 \%$ above poverty index" category in Australia [5]. Similar to these 
findings, our study found that pregnant women with household income $<$ S $\$ 2000 /$ month have increased likelihood of a poor diet quality. Diet quality may not be the priority of future mothers that are financially poorer. Pregnant women who are financially poorer might have less access to healthier foods due to lower income and/ or do not regard having a good diet as priority [36]. We also found that women with education levels of less than secondary were more likely to have a poorer diet quality. This is consistent with results from studies that found higher diet quality in women who had higher educational qualifications than those with lower educational qualifications $[15,33]$. Higher levels of education may be reflection of better nutritional knowledge and/or better socioeconomic status, which in turn are associated with a better overall diet quality [35].

In addition, we found that women with previous pregnancies were more likely to have a poorer diet quality compared to those with first-time pregnancies. This is comparable to previous findings showing better diet quality in nulliparous women than multiparous women $[5,34]$. This is likely due to an increased shift in focus from personal to fetal needs in first time pregnancies as compared to those with past pregnancies [37].

Previous associations of racial differences and diet quality during pregnancy reported differences in diet quality among people of varying ethnicity $[15,32]$. In our study, we found that Malay women were more likely to have a poorer diet quality compared to women of Chinese and Indian ethnicity. This could be related to socioeconomic circumstances as well as changes in diet due to cultural beliefs. Compared to the Chinese and Indian women, the Malay women in our cohort were reported previously to have the highest percent decrease in milk, fruit, rice, noodles and bread, and vegetables consumption during pregnancy than before pregnancy [38] and this would have inevitably affected diet quality. These two aforementioned associations are in line with a recent review, which also found that the quality of maternal diets varied across ethnicity and dependent on the number of offspring [39].

\section{Strengths of the HEI-SGP}

A strength of our study is the relatively large and diverse cohort within which HEI-SGP was examined. We have shown that the HEI-SGP can be used in a multi-ethnic cohort and we believe that the HEI-SGP is reproducible for use in other pregnant populations with similar ethnicities and dietary guidelines. Based on a one-day recall, the HEI-SGP gives a snapshot of the diet quality of the pregnant women. The key advantage is that it is simple, with easy to use components and methods to quantify. This may be useful as a screening tool to select women 'at risk' of poor diets for counselling/therapy and intervention.

\section{Limitations of the HEI-SGP}

It is recognised that a 24-hour recall reflects a day's intake and does not take into account day-to day variation or longer term dietary patterns, which may provide a more accurate assessment of diet quality. Participants were also asked to fast overnight prior the clinic visit when the $24 \mathrm{hr}$ recall was obtained and this could influence eating patterns of those who tend to eat very late at night.

The HEI-SGP was able to capture most but not all dietary constituents due to the lack of complete nutritional data on local foods e.g. sodium, "discretionary calories" from sugar. It is acknowledged that lack of these specific dietary constituents would thus preclude the ability of HEI-SGP to reflect diet quality with regards to these nutritional aspects of the diets. The HEI-SGP may also be limited because it does not directly capture overconsumption of certain food groups such as "total rice and alternatives", "total protein foods" or "dairy", which may contribute to excess pregnancy weight gain or an imbalance of macronutrients. However, it is important to consider that including too many components would add complexity and limit the use of the HEI-SGP for public health education and as a screening tool.

\section{Conclusions}

Our study has developed a diet quality tool, which has shown to quantitatively describe the quality of the diets of pregnant women in Singapore at 26-28 weeks gestation in the GUSTO study. This tool also provided a measure of the nutritional status of our cohort and identified maternal characteristics that are associated with poor diet and nutrition. Identifying 'at risk' characteristics will be important to guide public health policies that aim to promote maternal health and well-being. Although the present study was done on Singaporean women, the ethnic diversity in this study population suggests its application to other Asian populations. Validation of the HEISGP in other pregnant populations could provide valuable information for policy makers or nutrition educators in countries with similar multi-ethnic populations.

The HEI-SGP is a relatively simple, and easy to use method at a population level to identify women 'at risk' of poor diets for counselling/therapy and intervention at a population level. It could also potentially be adapted and incorporated into existing health screening tools.

\section{Additional file}

Additional file 1: A. Details of scoring a diet with the HEI-SGP. B Sub group analyses of maternal characteristics by Healthy Eating Index for pregnant women in Singapore (HEI-SGP) in the GUSTO cohort study. C. Foods included in Components of the Healthy Eating Index for pregnant women in Singapore. D. Components of the Healthy Eating Index for pregnant women in Singapore in comparison with HEl, AHEl, AHEI-P. (DOCX 33 kb) 


\begin{abstract}
Abbreviations
GUSTO: Growing up in Singapore towards healthy outcomes; A*Star: Agency for science, technology and research; NHS: National healthcare system; HEI-SGP: Healthy eating index for pregnant women in Singapore; HEl: Healthy eating index; USDA: United States Department of Agriculture; AHEI-P: Alternate healthy eating index for pregnancy; AHEl: Alternate healthy eating index; DQI-P: Dietary quality index for pregnancy; DQI-R: Diet quality index-revised; MDS: Mediterranean diet scale; MDS-P: Mediterranean diet scale for pregnant women; KKH: Kandang Kerbau Women's and Children's Hospital; NUH: National University Hospital; RDI: Recommended Dietary Intakes; BMI: Body mass index; FFQ: Food frequency questionnaire.
\end{abstract}

\section{Competing interests}

K. M. Godfrey and Y. S. Chong have received reimbursement for speaking at conferences sponsored by companies selling nutritional products. They are part of an academic consortium that has received research funding from Abbott Nutrition, Nestec and Danone. The other authors have no financial or personal competing interests.

\section{Authors' contribution}

MFFC and CYH designed the research. YSC, PDG, KMG, KK, and SMS designed and led the GUSTO study. MFFC and MC contributed to data collection. MFFC, MC and CYH contributed to data analysis. YHC and QPL provided statistical input. MFFC and CYH wrote the paper. MFFC and CYH have primary responsibility for the final content. All authors have read and approved the final manuscript.

\section{Acknowledgements}

The authors would like to thank the GUSTO study group which includes Pratibha Agarwal, Arijit Biswas, Choon Looi Bong, Birit FP Broekman, Shirong Cai, Jerry Kok Yen Chan, Yiong Huak Chan, Cornelia Yin Ing Chee, Helen Y. H Chen, Yin Bun Cheung, Audrey Chia, Amutha Chinnadurai, Chai Kiat Chng, Shang Chee Chong, Mei Chien Chua, Chun Ming Ding, Eric Andrew Finkelstein, Doris Fok, Marielle Fortier, Anne Eng Neo Goh, Yam Thiam Daniel Goh, Joshua J. Gooley, Wee Meng Han, Mark Hanson, Christiani Jeyakumar Henry, Joanna D. Holbrook, Chin-Ying Hsu, Hazel Inskip, Jeevesh Kapur, Kenneth Kwek, Ivy Yee-Man Lau, Bee Wah Lee, Yung Seng Lee, Ngee Lek, Sok Bee Lim, Yen-Ling Low, lliana Magiati, Lourdes Mary Daniel, Michael Meaney, Cheryl Ngo, Krishnamoorthy Naiduvaje, Wei Wei Pang, Anqi Qiu, Boon Long Quah, Victor Samuel Rajadurai, Mary Rauff, Salome A. Rebello, Jenny L. Richmond, Anne Rifkin-Graboi, Seang-Mei Saw, Lynette Pei-Chi Shek, Allan Sheppard, Borys Shuter, Leher Singh, Shu-E Soh, Walter Stunkel, Lin Lin Su, Kok Hian Tan, Oon Hoe Teoh, Mya Thway Tint, Hugo P S van Bever, Rob M. van Dam, Inez Bik Yun Wong, P. C. Wong, Fabian Yap, George Seow Heong Yeo.

\section{Funding}

This study is supported by the Translational Clinical Research (TCR) Flagship Program on Developmental Pathways to Metabolic Disease funded by the National Research Foundation (NRF) and administered by the National Medical Research Council (NMRC), Singapore- NMRC/TCR/004-NUS/2008. Additional funding is provided by the Singapore Institute for Clinical Sciences, A*STAR and Nestec. K. M. Godfrey is supported by the National Institute for Health Research through the NIHR Southampton Biomedical Research Centre.

\section{Author details}

'Department of Nutrition and Dietetics, Flinders University of South Australia, Sturt Rd, Bedford Park, SA 5042, Australia. ${ }^{2}$ Department of Dietetics and Nutrition, Ng Teng Feng General Hospital, Jurong Health Services, 1 Jurong East St 21, 5609606 Singapore, Singapore. ${ }^{3}$ Singapore Institute for Clinical Sciences, Brenner Centre for Molecular Medicine 30 Medical Drive, S117609 Singapore, Singapore. ${ }^{4}$ Biostatistics Unit, National University of Singapore, 21 Lower Kent Ridge Rd, S119077 Singapore, Singapore. ${ }^{5}$ Medical Research Council Lifecourse Epidemiology Unit and NIHR Southampton Biomedical Research Centre, University of Southampton and University Hospital Southampton NHS Foundation Trust, University Rd SO17 1BJ, Southampton, UK. ${ }^{6}$ Division of Obstetrics and Gynaecology, Kandang Kerbau Women's and Children's Hospital, 100 Bukit Timah Rd, S229899 Singapore, Singapore. ${ }^{7}$ Saw Swee Hock School of Public Health, National University of Singapore and National University Health System, 12 Science Drive 2 S117549, Singapore, Singapore. ${ }^{8}$ Liggins Institute, University of Auckland, Building 505, 85 Park
Road, Grafton, Auckland, New Zealand. ${ }^{9}$ Department of Obstetrics \& Gynaecology, Yong Loo Lin School of Medicine, National University of Singapore and National University Health System, 10 Medical Drive, S117597 Singapore, Singapore. ${ }^{10}$ Department of Paediatrics, Yong Loo Lin School of Medicine, National University of Singapore and National University Health System, 10 Medical Drive, S117597 Singapore, Singapore.

\section{Received: 2 September 2015 Accepted: 3 November 2015 Published online: 25 November 2015}

\section{References}

1. Kourlaba G, Panagiotakos DB. Dietary quality indices and human health: a review. Maturitas. 2009:62(1):1-8.

2. Wirt A, Collins CE. Diet quality-what is it and does it matter? Public Health Nutr. 2009;12(12):2473-92.

3. Shahril MR, Sulaiman S, Shaharudin SH, Akmal SN. Healthy eating index and breast cancer risk among Malaysian women. Eur J Cancer Prev. 2013;22(4):342-7.

4. Li S, Chiuve SE, Flint A, Pai J, Forman JP, Hu FB, et al. Better Diet Quality and Decreased Mortality Among Myocardial Infarction Survivors. JAMA Int Med. 2013;173:1808-18.

5. Bodnar LM, Siega-Riz AM. A Diet Quality Index for Pregnancy detects variation in diet and differences by sociodemographic factors. Public Health Nutr. 2002;5(6):801-9.

6. Tsigga M, Filis V, Hatzopoulou K, Kotzamanidis C, Grammatikopoulou MG Healthy Eating Index during pregnancy according to pre-gravid and gravid weight status. Public Health Nutr. 2011;14(2):290-6.

7. Barker DJ. The origins of the developmental origins theory. J Intern Med. 2007:261(5):412-7.

8. Kaiser L, Allen LH. Position of the American Dietetic Association: nutrition and lifestyle for a healthy pregnancy outcome. J Am Diet Assoc. 2008; 108(3):553-61.

9. Pregnancy and diet: Health Promotion Board Singapore; 2013 [cited 2013 August]. Available from: http://www.hpb.gov.sg/HOPPortal/health-article/3826.

10. Jang YA, Lee HS, Kim BH, Lee Y, Lee HJ, Moon JJ, et al. Revised dietary guidelines for Koreans. Asia Pac J Clin Nutr. 2008;17 Suppl 1:55-8.

11. FSANZ. Pregnancy and healthy eating: Food Standards Australia New Zealand (FSANZ); 2011 [cited 2013 Dec]. Available from: http://www.foodstandards.gov. au/consumer/generalissues/pregnancy/Pages/defaultaspx.

12. Moran $L$, Sui Z, Cramp CS, Dodd JM. A decrease in diet quality occurs during pregnancy in overweight and obese women which is maintained post-partum. Int J Obes (Lond). 2013;37(5):704-11.

13. Pick ME, Edwards M, Moreau D, Ryan EA. Assessment of diet quality in pregnant women using the Healthy Eating Index. J Am Diet Assoc. 2005;105(2):240-6.

14. Kennedy ET, Ohls J, Carlson S, Fleming K. The Healthy Eating Index: design and applications. J Am Diet Assoc. 1995;95(10):1103-8.

15. Rifas-Shiman SL, Rich-Edwards JW, Kleinman KP, Oken E, Gillman MW. Dietary quality during pregnancy varies by maternal characteristics in Project Viva: a US cohort. J Am Diet Assoc. 2009;109(6):1004-11.

16. McCullough ML, Feskanich D, Stampfer MJ, Giovannucci EL, Rimm EB, Hu FB, et al. Diet quality and major chronic disease risk in men and women: moving toward improved dietary guidance. Am J Clin Nutr. 2002;76(6):1261-71.

17. Haines PS, Siega-Riz AM, Popkin BM. The Diet Quality Index Revised. J Am Diet Assoc. 1999;99(6):697-704.

18. Mariscal-Arcas M, Rivas A, Monteagudo C, Granada A, Cerrillo I, Olea-Serrano F. Proposal of a Mediterranean diet index for pregnant women. Br J Nutr. 2009:102(5):744-9.

19. Trichopoulou A, Costacou T, Bamia C, Trichopoulos D. Adherence to a Mediterranean diet and survival in a Greek population. N Engl J Med. 2003; 348(26):2599-608.

20. Soh SE, Tint MT, Gluckman PD, Godfrey KM, Rifkin-Graboi A, Chan YH, et al. Cohort Profile: Growing Up in Singapore Towards healthy Outcomes (GUSTO) birth cohort study. Int J Epidemiol. 2013;43(5):1401-9.

21. Conway JM, Ingwersen LA, Vinyard BT, Moshfegh AJ. Effectiveness of the US Department of Agriculture 5-step multiple-pass method in assessing food intake in obese and nonobese women. Am J Clin Nutr. 2003:77(5):1171-8.

22. HPB. Food Info Search Singapore: Health Promotion Board; 2012 [updated September 2012; cited 2013 Jun ]. Available from: http://www.hpb.gov.sg/ HOPPortal/health-article/HPBSUEXTAPP1_4021885.

23. Guenther PM, Reedy J, Krebs-Smith SM. Development of the Healthy Eating Index-2005. J Am Diet Assoc. 2008;108(11):1896-901. 
24. Guenther PM, Casavale KO, Reedy J, Kirkpatrick SI, Hiza HA, Kuczynski KJ, et al. Update of the Healthy Eating Index: HEl-2010. J Acad Nut Diet. 2013; 113(4):569-80

25. Forsum E, Lof M. Energy metabolism during human pregnancy. Annu Rev Nutr. 2007;27:277-92.

26. Health-promotion-Board. Recommended Dietary Allowances Singapore: Health promotion board; 2014 [cited 2014 March]. Available from: http:// www.hpb.gov.sg/HOPPortal/health-article/2652

27. Kopp-Hoolihan LE, van Loan MD, Wong WW, King JC. Longitudinal assessment of energy balance in well-nourished, pregnant women. Am J Clin Nutr. 1999:69(4):697-704.

28. Guenther PM, Reedy, J., Krebs-Smith, S.M., Reeve, B.B., \& Basiotis, P.P. Healthy Eating Index-2005 Technical Report2007 [cited 2013 july ]. Available from: http://www.cnpp.usda.gov/HealthyEatinglndex.htm

29. Board Hp. My Healthy Plate Singapore Singapore2014 [cited 201414 July]. Available from: http://www.hpb.gov.sg/HOPPortal/health-article/HPB064355.

30. Vitamins and Nutrition in Pregnancy U.K. : National Health Service; 2013 [cited 2013 August]. Available from: http://www.nhs.uk/Conditions/pregnancy-andbaby/pages/vitamins-minerals-supplements-pregnant.aspx\#close.

31. Qiu C, Frederick IO, Zhang C, Sorensen TK, Enquobahrie DA, Williams MA. Risk of gestational diabetes mellitus in relation to maternal egg and cholesterol intake. Am J Epidemiol. 2011;173(6):649-58.

32. Mantzoros CS, Sweeney L, Williams CJ, Oken E, Kelesidis T, Rifas-Shiman SL, et al. Maternal diet and cord blood leptin and adiponectin concentrations at birth. Clin Nutr. 2010;29(5):622-6.

33. Chatzi L, Mendez M, Garcia R, Roumeliotaki T, Ibarluzea J, Tardon A, et al. Mediterranean diet adherence during pregnancy and fetal growth: INMA (Spain) and RHEA (Greece) mother-child cohort studies. Br J Nutr. 2012; 107(1):135-45

34. Laraia BA, Bodnar LM, Siega-Riz AM. Pregravid body mass index is negatively associated with diet quality during pregnancy. Public Health Nutr. 2007;10(9):920-6.

35. Fowles ER, Bryant M, Kim S, Walker LO, Ruiz RJ, Timmerman GM, et al. Predictors of dietary quality in low-income pregnant women: a path analysis. Nurs Res. 2011:60(5):286-94.

36. Watts $V$, Rockett $H$, Baer H, Leppert J, Colditz G. Assessing diet quality in a population of low-income pregnant women: a comparison between Native Americans and whites. Matern Child Health J. 2007;11(2):127-36.

37. Darvill $R$, Skirton H, Farrand P. Psychological factors that impact on women's experiences of first-time motherhood: a qualitative study of the transition. Midwifery. 2010;26(3):357-66

38. Chen LW, Low YL, Fok D, Han WM, Chong YS, Gluckman P, et al. Dietary changes during pregnancy and the postpartum period in Singaporean Chinese, Malay and Indian women: the GUSTO birth cohort study. Public Health Nutr. 2013;17:1-9.

39. Inskip H, Baird J, Barker M, Briley AL, D'Angelo S, Grote V, et al. Influences on adherence to diet and physical activity recommendations in women and children: insights from six European studies. Ann Nutr Metab. 2014;64(3-4):332-9.

\section{Submit your next manuscript to BioMed Central and take full advantage of:}

- Convenient online submission

- Thorough peer review

- No space constraints or color figure charges

- Immediate publication on acceptance

- Inclusion in PubMed, CAS, Scopus and Google Scholar

- Research which is freely available for redistribution 